\title{
Brauchen wir noch Psychotherapieschulen bzw. -verfahren?
}

Prof. Dr. Mathias Berger ist Direktor der Klinik für Psychiatrie und Psychotherapie an der Universität Freiburg, Herausgeber eines der großen Psychiatrielehrbücher und war bis vor einiger Zeit Präsident der Deutschen Gesellschaft für Psychiatrie, Psychotherapie und Nervenheilkunde (DGPPN). An der Freiburger Klinik hat er aktiv Untersuchungen zur Psychotherapie gefördert, einerseits zu unterschiedlichen Krankheitsbildern, andererseits zu neuen Psychotherapieformen. So sind die Entwicklungen der IPT (Interpersonelle Psychotherapie der Depression), der DBT (Dialektisch-behaviorale Therapie), der Schematherapie oder des CBASP (Cognitive Behavioral Analysis System of Psychotherapy) eng mit der Freiburger Klinik verknüpft.

Prof. Dr. Michael Linden ist Arzt für Psychiatrie und Psychosomatik und Psychologischer Psychotherapeut. Er leitet eine Klinik für Verhaltenstherapie bei Berlin, ist Mitglied des wissenschaftlichen Beirats Psychotherapie und Sprecher des Referats Psychotherapie der DGPPN. Er hat umfangreiche Studien zur Prozessforschung, Ergebnisevaluation, Qualitätssicherung und Versorgungsforschung im Bereich Psychotherapie publiziert und ist unter anderem Herausgeber der Zeitschrift VerHALTENSTHERAPIE und des Verhaltenstherapie-Manuals.

\section{+ Pro}

Psychotherapie ohne Psychotherapieschulen ist grundsätzlich nicht möglich

Definitorische Vorbemerkungen

Psychotherapie ist eine Form zwischenmenschlicher Begegnung, die abzugrenzen ist von anderen Begegnungs- und Hilfsangeboten wie allgemein menschlicher Zuwendung, Ehe-, Berufs- und Erziehungsberatung, Manager-Training und vielem anderem mehr. Bestimmungsmerkmale der Psychotherapie sind, dass es sich dabei um eine theoriegeleitete, zielgerichtete und evidenzbasierte Beeinflussung von körperlichen und seelischen Krankheitsprozessen durch die Interaktion mit einem approbierten Psychotherapeuten handelt. Sie muss daher auch lehr- und lernbar sowie qualitätsgesichert und gesetzlich normiert sein [Helmchen et al., 1982; Gemeinsamer Bundesausschuss, GeBA, 2007].

In der langen Anwendungs- und Forschungstradition der Psychotherapie wurden eine Reihe unterschiedlicher Psychotherapieschulen, d.h. psychotherapeutischer Grundorientierungen entwickelt. Dies sind störungsübergreifende Theoriesysteme, die es erlauben, menschliches Verhalten und insbesondere psychische Erkrankungen zu beschreiben, zu verstehen und darauf abgestellte Behandlungsinterventionen $\mathrm{zu}$ operationalisieren und kontrolliert anzuwenden. Den Grundorientierungen können in Anlehnung an das deutsche Psychotherapeutengesetz [PsychThG, 1998] Psychotherapieverfahren zugeordnet werden, die als ausbildungsorientierte Operationalisierungen der Grundorientierungen verstanden werden können. Ein Psychotherapieverfahren ist das, was ein Therapeut lernen bzw. können muss, um als «Ärztlicher» oder «Psychologischer» Psychotherapeut arbeiten zu dürfen. Psychotherapieverfahren stellen Erklärungsmodelle für verschiedenste psychische Störungen zur Verfügung und beschreiben daraus abgeleitete Therapietechniken (z.B. in der VT: Exposition, kognitives Rehearsal), Therapiemodule, die mehrere Techniken umfassen (z.B. in der VT: AssertivenessTraining) oder störungsorientierte Therapieprogramme bzw. - nach Diktion des GeBA und der Pychotherapierichtlinien [GeBA, 2007] - Behandlungsstrategien (z.B. in der VT: dialektische Verhaltenstherapie).

Es ist gesetzlich vorgegeben, dass Psychotherapeuten in Verfahren ausgebildet und verfahrensbezogen approbiert werden. Die Ausbildung in einem Psychotherapieverfahren bzw. die theoretischen Modelle und das methodische Repertoire eines Verfahrens müssen es ermöglichen, einen wesent-

\section{KARGER}

Fax +497614520714

Information@Karger.de

www.karger.com
(C) 2009 S. Karger GmbH, Freiburg 
lichen Teil des heterogenen Spektrums psychotherapiepflichtiger psychischer Erkrankungen adäquat zu behandeln. Damit ein Verfahren als Ausbildungsgrundlage anerkannt werden kann, muss daher nach den Regeln des Wissenschaftlichen Beirats Psychotherapie [WBP, 2009] belegt sein, dass es bei unterschiedlichen Störungen aus Kapitel F der ICD-10 einsetzbar und wirksam ist. Derartige Psychotherapieverfahren sind z.B. Verhaltentherapie, Psychodynamische Psychotherapie, Klientenzentrierte Psychotherapie, Gestaltpsychotherapie oder Systemische Psychotherapie, die jedoch noch nicht alle im Rahmen des Gesundheitswesens der Bundesrepublik Deutschland wissenschaftlich anerkannt sind.

Neben den Psychotherapieverfahren gibt es nach den Psychotherapierichtlinien noch Psychotherapiemethoden [GeBA, 2007]. Psychotherapiemethoden sind therapeutische Verfahren mit technisch und theoretisch begrenzter Gültigkeit und eingeschränktem Indikationsspektrum. Eine Ausbildung in einer Psychotherapiemethode (z.B. neuropsychologische Psychotherapie) führt nicht zur Approbation als Psychologischer Psychotherapeut oder einer Facharztanerkennung, jedoch setzt deren Anwendung die Approbation bzw. Weiterbildung in einem wissenschaftlich anerkannten Psychotherapieverfahren voraus.

Im Folgenden werden einige Argumente angeführt, warum eine Psychotherapie ohne psychotherapeutische Grundorientierung («Schule») bzw. ohne Psychotherapieverfahren grundsätzlich nicht möglich ist und warum eine «integrative Psychotherapie» oder eine nur «störungsspezifisch» definierte Psychotherapie auch nicht wünschenswert sind. Diese Argumente leiten sich ab aus der Wissenschaftstheorie, aus Erfordernissen der Störungs- und Therapiekonzeptualisierung, aus Ausbildungsnotwendigkeiten, aus Gesichtspunkten der Qualitätssicherung und des Patientenschutzes und nicht zuletzt aus Überlegungen zur Organisation des Gesundheitswesens.

\section{Wissenschaftstheoretische Überlegungen}

Psychotherapieschulen sind Theoriesysteme, die Begriffe und Regeln vorgeben zur Beschreibung, Erklärung und Veränderung von psychischen Krankheiten. Psychotherapeuten können ohne Theorien nicht arbeiten. Es hat noch nie jemand ein «Schema» oder «Widerstand» gesehen. Wissenschaftstheoretisch gesprochen sind dies hypothetische Konstrukte, die aus übergeordneten Theorien abgeleitet werden [Groeben und Westmeyer, 1975]. Wer solche Begriffe verwendet, muss auch die Theorie anwenden, in die sie eingebettet sind. Wer im Kontext von Verhaltenstherapie also von «Widerstand» spricht, wenn ein Patient nicht tut, was er geheißen wurde, beweist damit unzureichende Fachkenntnis. Er könnte sagen, dass der Patient seine Hausaufgaben nicht erwartungsgemäß durchgeführt hat (Beschreibungsebene) und dann eine Verhaltensanalyse machen (Interpretationsebene im Theoriesystem der Verhaltenstherapie). Eine Interpretation mithilfe des hypothetischen Konstrukts «Widerstand» setzt hingegen die Einordnung in eine psychodynamische Theorie und eine Übertragungs- und Widerstandsanalyse voraus. Hypothe- tische Konstrukte aus ihrem theoretischen Bezugsrahmen herauszunehmen wird in der Wissenschaftstheorie als «Reifizierung» und als Fehler beschrieben. Es gereicht in der Wissenschaft wie im richtigen Leben nicht zur Ehre, wenn man Fremdwörter falsch benutzt.

Insofern ist wissenschaftlich auch nicht nachvollziehbar, was mit der Forderung gemeint sein soll, aus unterschiedlichen Schulen «das Beste» in einer «integrativen» Psychotherapie zusammenzufassen. Ein psychotherapeutisches Esperanto? Ebensowenig ist einsichtig, warum man einer unitheoretischen Welt das Wort redet. Dazu ist zum Ersten anzumerken, dass die Theorien, die sich als «allgemeine» oder «integrative» Psychotherapie [Norcross und Goldstein, 1992; Grawe, 1995; Sponsel, 1995; Petzold und Märtens, 1998] bezeichnen und damit implizit Anspruch auf universelle Gültigkeit erheben, wiederum nichts anderes sind als weitere Theoriesysteme unter anderen. Ihre empirische und technische Ausarbeitung ist jedoch weit davon entfernt, die Ansprüche an ein Psychotherapieverfahren auch nur im Ansatz zu erfüllen. Sonst könnten ihre Vertreter ja die Anerkennung als Verfahren beantragen. Bei näherer Prüfung handelt es sich zudem weitgehend um Varianten bestehender psychotherapeutischer Grundorientierungen ohne grundsätzlichen Neuigkeitswert, also um längst Bekanntes, nur anders ausgedrückt [Rogers, 1961; Fromm, 1974; Brumund und Märtens, 1998].

Zum Zweiten können Theorien grundsätzlich nicht wahr oder falsch sein, sondern sind nach ihrer Widerspruchsfreiheit, Einfachheit und prognostischen Utilität zu beurteilen. Deshalb kann man dieselben Sachverhalte unter Anwendung unterschiedlicher Theorien beschreiben. Die Wahlmöglichkeit zwischen unterschiedlichen Theorien zur Erklärung und zum Umgang mit demselben Sachverhalt hat nicht nur in der Physik, sondern erst recht in den psychotherapeutischen Wissenschaften große Vorteile. Sie eröffnet alternative Erklärungszugänge und damit auch Therapieoptionen und ermöglicht nicht zuletzt auch Therapeuten, die Therapieform zu lernen, die ihnen persönlich am meisten liegt.

\section{Störungs- und Therapiekonzeptualisierung}

Theorien werden unter anderem nach ihrer Utilität beurteilt. Krankheitsmodelle sind dann gut, wenn sie Handlungsempfehlungen und Vorhersagen ermöglichen. Allerdings sind auch die Interventionsbegriffe (z.B. Exposition) hypothetische Konstrukte. Wer von Exposition spricht, muss die Theorie der Konditionierung und Attribution und alle daraus abgeleiteten Regeln mitdenken. Daraus folgen theorieeingebettet sogenannte «Operationalisierungen» der hypothethischen Konstrukte. «Exposition» heißt also nicht, den Patienten «mit Angst in die U-Bahn zu schicken, damit die Angst nachlässt». So denken sich das gelegentlich Amateure. Korrekt ist es, unter Bezug auf das Begriffssystem der verhaltenstherapeutischen Grundorientierung mit der daraus abgeleiteten Technik der Verhaltensanalyse ein individuelles Störungsmodell zu erarbeiten und dann, je nach Fall, eine Stimulushabituierung, 
ein kognitives Reframing oder ein Reaktionsmanagement durchzuführen, was übrigens wieder zu operationalisierende hypothethische Konstrukte sind. Es ist also schwer nachzuvollziehen, was ein Tiefenpsychologe meint, wenn er angibt, im Rahmen seiner Behandlung auch eine Exposition zu machen.

Psychotherapeutische Verfahren beschreiben unter Bezug auf eine theoretische Grundorientierung die Art der Gestaltung der therapeutischen Beziehung, störungsübergreifend einzusetzende Basistechniken, störungsspezifische Interventionen, Strukturierungsvorgaben für den therapeutischen Prozess in der Stunde wie im gesamten Therapieverlauf und schließlich heuristische Modelle für unterschiedliche Störungen [Linden et al., 2007a]. Besonders wichtig sind die schulenspezifischen Interventionen, die störungsübergreifend zum Einsatz kommen, also die Basistechniken. So wird jeder Verhaltenstherapeut in jeder Therapie Hausaufgaben stellen oder automatische Gedanken analysieren, was in der Psychoanalyse oder der Klientenzentrierten Psychotherapie so sicher nicht vorkommt [Linden et al., 2007b]. Auf der Ebene der Basistechniken lassen sich Schulen sehr präzise voneinander unterscheiden. Fallbeschreibungen und -erklärungen von Therapieanträgen im Richtlinienverfahren lesen sich ganz unterschiedlich, je nach Schule des Therapeuten.

Hinzu kommt, dass Änderungsprozesse in einer Psychotherapie wesentlich auch dadurch angestoßen werden, dass der Patient sich, seine Welt und seine Störung neu zu verstehen lernt und daraus neue Bewältigungs- oder Erlebensmöglichkeiten ableiten kann. Auch Patienten benötigen daher Begriffssysteme und theoretische Modelle. Analysiert man Misserfolge und Nebenwirkungen von Therapien, zeigt sich regelmäßig, dass uneindeutige Modelle vermittelt und gültige Operationalisierungen missachtet wurden. Patienten werden z.B. «exponiert», ohne dies auch nur ansatzweise verhaltensanalytisch zu planen und anzuleiten, mit dem Ergebnis, dass es ihnen nachher schlechter geht als vorher [Lieberei und Linden, 2008].

Natürlich schließt ein Verfahrensbezug ein störungsspezifisches Vorgehen nicht aus. Selbstverständlich gibt es für unterschiedliche Störungen innerhalb eines Verfahrens unterschiedliche Modelle. Keine Grundorientierung erklärt z.B. eine Zwangserkrankung nach demselben Modell wie eine Depression. Selbst für dieselbe Störung finden sich in der Regel mehrere Erklärungsmodelle. So sind z.B. in der Verhaltenstherapie bis zu einem Dutzend unterschiedlicher Erklärungsmodelle für Zwangsstörungen beschrieben worden, was es dem Therapeuten ermöglicht, für seinen Patienten das Modell auszuwählen, das den besten Erklärungs- und Vorhersagewert hat und am wenigsten Exhaurierungen erfordert. Es gibt also nicht nur störungsspezifische, sondern auch noch patienten(!)spezifische Adaptationen innerhalb eines Verfahrens. Dennoch benötigen alle diese störungsspezifischen oder Individualmodelle als Referenz übergeordnete Theorien, eben psychotherapeutische Grundorientierungen und Verfahren und daraus abgeleitete Operalisierungen von Interventionen.

\section{Ausbildungsnotwendigkeiten}

Psychotherapieverfahren stellen per definitionem Erklärungsmodelle und Interventionsoptionen für ein breites Spektrum an Störungen zur Verfügung Daher ist die Ausbildung in einem Verfahren hinreichend, um als Psychotherapeut arbeiten zu können. Eine verfahrensbezogene Ausbildung vermittelt die erforderlichen Grundkompetenzen, um unterschiedlichste Fälle behandeln zu können [GeBA, 2007; WBP, 2009]. Im Vergleich zur Musik könnte man sagen, dass eine verfahrensbezogene Ausbildung wie die Ausbildung an einem Instrument ist. Wer erst einmal Klarinette an sich spielen kann, kann dann auch ohne größere Probleme unterschiedliche Stücke spielen. Es gibt keine «stückespezifische» musikalische Ausbildung. Mit stückespezifischen Besonderheiten befasst man sich erst, wenn man das Instrument an sich beherrscht. Genauso gilt, dass ein Therapeut dann ein Verhaltenstherapeut ist, wenn er über die Grundkompetenz verfügt, eine Verhaltensanalyse zu machen, Hausaufgaben zu stellen, Kognitionen zu analysieren oder Problemlösungen zu strukturieren. Er kann dann bei Persönlichkeitsstörungen dem kognitiven Modell folgend Schemata analysieren und verändern oder dem Coping-Modell entsprechend soziale Kompetenz trainieren oder dem Emotionsmodell gemäß Achtsamkeitsübungen durchführen. Tiefenpsychologen würden bei demselben Patienten mit Übertragungsanalysen beginnen, mit Triangulation fortfahren usw. Unter welchen Bedingungen welches Vorgehen zu welchen Ergebnissen führt und ob dies ex ante vorherzusagen ist, ist wissenschaftlich zu untersuchen.

Eine störungsspezifische Ausbildung ist auch aus Gründen der Ausbildungsorganisation nicht möglich. Wenn ein Ausbildungs- oder Weiterbildungskandidat bis zu seiner Abschlussprüfung ein Dutzend unterschiedlicher Patienten behandelt hat, dann war er fleißig, ist aber weit davon entfernt, auch nur einen Bruchteil der von ihm später zu behandelnden Probleme und Störungen gesehen zu haben. Außerdem würde es auch nicht helfen, einmal einen Zwang, eine Depression, eine Borderline-Störung usw. behandelt $\mathrm{zu}$ haben, um dies anschließend kompetent zu können. Es bleibt also nichts anderes übrig, als die beschriebene Ausbildung in verfahrensbezogenen Basiskompetenzen.

Wenn unterschiedliche parallele oder komplementäre Theoriesysteme wünschenswert sind, dann ist die Frage, ob Therapeuten nicht in möglichst vielen oder gar allen verfügbaren Verfahren ausgebildet werden sollten. Korrekt muss die Frage lauten, ob dies möglich ist. Theorien sind wie Sprachen. Der eigenen Erfahrung nach ist es besser, eine Sprache möglichst intensiv zu lernen, als von vielen jeweils ein wenig kennenzulernen. So kommt wohl auch niemand auf die Idee, einen Sprachkurs von 100 Stunden in Blöcken à 5 auf Englisch, Französisch, Russisch, Japanisch usw. zu verteilen, anstatt alle Stunden auf eine Sprache zu verwenden, welche auch immer das sei.

Eine oberflächliche Befassung mit unterschiedlichen Theoriesystemen birgt in der Psychotherapie auch Gefahren. Die Kandidaten verwechseln die Verwendung unsauber defi- 
nierter Fremdwörter schnell mit Fachkompetenz. Die Reifizierung hypothetischer Konstrukte und die begriffliche Beliebigkeit sind in der Psychotherapie umso weiter verbreitet, je geringer die Fachkenntnisse sind (siehe «Widerstand» im Mund eines Verhaltenstherapeuten oder «Exposition» bei Tiefenpsychologen). Der schwadronierende Umgang mit Begriffen ist ein Charakteristikum von Amateurpsychotherapeuten am Stammtisch. Werden diese an Patienten gelassen, wird es gefährlich, denn die Psychotherapie ist ein Feld, das anfällig ist für Scharlatanerie und Selbstüberschätzung.

\section{Qualitätssicherung und Organisation des Gesundheitswesens}

Ein konstitutives Merkmal von Psychotherapie ist, dass sie heilkundlichen gesetzlichen Normierungen unterliegt. Dazu gehört als erstes, dass nicht jeder Einfall eines Therapeuten als Psychotherapie zählt. Heilkundliche Psychotherapie ist auf wissenschaftlich anerkannte Verfahren begrenzt, und das ist gut so. In der Berliner Stadtzeitung Zitty wird beispielsweise «Zauber-Psychotherapie» annonciert. Ein Patient muss die Sicherheit haben, davon verschont zu werden, wenn er einen approbierten Psychotherapeuten aufsucht, und dies wird gelegentlich auch gerichtlich geprüft.

Eine Frage ist, wie mit Neuentwicklungen umzugehen ist, wie beispielsweise der IPT [Schramm, 1998], dem CBASP [McCullough, 2006], der DBT [Linehan, 1993] oder der Weisheitstherapie [Schippan et al., 2004]. Zunächst einmal ist es die Aufgabe der Autoren selbst zu sagen, worum es sich bei ihren Entwürfen handelt. Wenn es sich um ein Therapieprogramm im Rahmen einer bestehenden Grundorientierung handelt, so ist dies mit Bezug auf die jeweiligen Theoriesysteme und Basistechniken zu zeigen, was beispielsweise für die DBT als Therapieprogramm in der Verhaltenstherapie nach Vorlage im WBP erfolgte. Die Anwendung von DBT setzt demnach eine Ausbildung in Verhaltenstherapie voraus und kann damit als verhaltenstherapeutische Behandlungsstrategie mit spezieller Indikation bei Borderline-Störungen verstanden werden [GeBA, 2007]. Ist keine Zuordnung zu einem bestehenden Verfahren möglich, weil der neue Ansatz eine eigene theoretische Basis hat und hinreichend breit einsetzbar ist, so ist zu prüfen, ob es sich um eine eigene Grundorientierung bzw. ein eigenes Verfahren handelt. Dazu sind die oben genannten Anforderungen zu erfüllen. Gelingt das nicht, dann wäre bei unabhängiger Theoriebasis zu prüfen, ob die Voraussetzungen für die Anerkennung als Methode gegeben sind, was eine Anwendung - gegebenenfalls nach spezieller Weiterbildung - durch jeden approbierten Psychotherapeuten ermöglichen würde. Was nicht sein darf, ist, dass Protagonisten bestimmter Psychotherapievarianten verlangen, dass ihr Lieblingsvorgehen am Patienten zur Anwendung kommt und gar noch von den Kassen bezahlt wird, ohne dass eine entsprechende Klärung erfolgt ist. Wenn hinreichende theoretische Vorarbeiten und empirische Evidenzen vorliegen, sollten die vorgenannten Klärungen keine Probleme darstellen.
Die Einordnung einer Psychotherapie als Verfahren, als Behandlungsstrategie innerhalb eines Verfahrens oder als unabhängige Methode ist Aufgabe des WBP. Damit ist nicht gleichzeitig entschieden, dass die Krankenversicherungen auch die Kosten für neue Therapievarianten übernehmen müssen, mögen sie so wirksam sein, wie sie wollen. Die Entscheidung über die Kostenübernahme obliegt dem GeBA, der seinen Beschlüssen differenzierte versorgungspolitische Überlegungen zugrunde legt. Aspirin ist bei Schmerzen unbestreitbar wirksam und wird von den Krankenkassen dennoch selten erstattet. Für Psychotherapie gelten keine Sonderregeln. So werden für die Klientenzentrierte Gesprächspsychotherapie trotz wissenschaftlicher Anerkennung als Verfahren keine Kosten übernommen, und die IPT müsste überhaupt erst einmal ihren Status klären, bevor auch nur daran zu denken wäre.

Neben der inhaltlichen Eingrenzung der heilkundlichen Psychotherapie ist auch ihre Anwendung an gesetzliche Voraussetzungen geknüpft. Nicht jeder darf Psychotherapie im Sinne einer Krankenbehandlung anwenden, sondern er oder sie muss staatlich approbiert sein. Die Approbation ist jedoch nur eine Eintrittsqualifikation. Für bestimmte Behandlungen werden zusätzliche Fachkundenachweise gefordert. Im deutschen Gesundheitswesen beginnt dies mit der bei Ärzten zwingend geforderten Facharztqualifikation und endet damit, dass z.B. ein bescheinigter, mehrtägiger Kurs verlangt wird, um einen Antrag auf Einleitung einer Rehabilitationsmaßnahme ausfüllen zu dürfen. Für mögliche Fachkundenachweise gibt es also keine Grenzen. Die Frage ist, auf welcher Ebene in der Psychotherapie spezifische Fachkundenachweise verlangt werden sollten. Derzeit ist dies die verfahrensbezogene Ausoder Weiterbildung. Man geht nicht davon aus, dass jemand nach einem Psychologie- oder Medizinstudium die Fachkunde hat, um Psychotherapie erbringen zu können. Auch allgemeine Psychotherapiekenntnisse, wie sie möglicherweise im Studium erworben wurden, oder die Approbation reichen nicht aus, sondern erst eine mehrjährige, curricular organisierte und staatlich überwachte und abgeprüfte Aus- oder Weiterbildung in einem definierten wissenschaftlich anerkannten Verfahren.

Würde der Verfahrensbezug ersetzt durch jeweils gesonderte Fachkundenachweise für Therapiemethoden und -strategien oder gar -module und -techniken, dann müsste in $\mathrm{Zu}$ kunft jeder Psychotherapeut gesondert Scheine machen für Neuropsychologie, Schematherapie, IPT, DBT, CBASP usw., bevor er diese Therapievarianten anwenden dürfte. Würde man dies noch mit einem engen Störungsbezug verknüpfen, dann müsste jeder Psychotherapeut zusätzlich Scheine machen für Borderline, Dysthymie, rezidivierende Depression, Zwang etc., bevor er sie behandeln dürfte. - Die Perspektive lässt erschaudern, steht aber bevor. So wurde bereits ernsthaft vorgetragen, dass organische Psychosyndrome künftig nur noch von Therapeuten mit einer (schein-)bestätigten Zusatzqualifikation in Neuropsychologie behandelt werden dürften. Dies hätte zur Folge, dass die meisten Psychotherapeuten, Psychiater oder Neurologen einen Demenzkranken oder 
einen Patienten nach zerebralem Insult ohne Zusatzweiterbildung nicht mehr behandeln dürften. Ähnliche Forderungen gibt es auch für andere Therapieformen (Schematherapie, DBT, IPT, EMDR usw.).

Ginge es hierbei nur um Auseinandersetzungen zwischen Wissenschaftlern und Therapie-Gurus oder um Copyright-Ansprüche einzelner Autoren, könnte man diese Diskussionen mit Gelassenheit beobachten. Die Entwicklung ist jedoch ernstzunehmen, weil im Hintergrund die Kostenträger stehen (vertreten u.a. durch den GeBA), die diese Entwicklung aufmerksam verfolgen und offensichtlich fördern. Leitende Repräsentaten des GeBA haben bereits im Deutschen Ärzteblatt geäußert, dass ein Fachkundenachweis für unterschiedliche Psychotherapiemethoden künftig wohl unvermeidbar sei. Das vordergründige Argument für diese Forderung ist die Qualitätssicherung. Allerdings ist bislang mit keiner einzigen Zahl belegt, dass ein Zusatzschein in CBASP, DBT usw. zu besseren Therapieergebnissen führt als eine Grundausbildung in Verhaltenstherapie mit kontinuierlicher Fortbildung und Kenntnisnahme der wenigen neuen Ideen in den Konzepten von McCullough, Linehan etc. Fakt und gut belegt ist hingegen, dass Fachkundenachweise eines der besten Mittel zur Leistungs- und damit Kostenbegrenzung sind. Daher sind sie für Kostenträger sehr attraktiv, auch wenn sie dem Patienten nicht viel bringen. Für eine wirksame Qualitätssicherung sind andere Instrumente erforderlich.

Wer also künftig einen eigenen Schein zur Voraussetzung für jeden psychotherapeutischen Handschlag machen möchte, sollte sich aktiv für das Konstrukt störungsspezifischer Psychotherapiemethoden einsetzen. Dies ist fachlich zwar überflüssig, hat aber aus Sicht der «Vertreter» der verschiedenen Therapievarianten fraglos den Vorteil, dass dann auch approbierte Kolleginnen und Kollegen, natürlich gegen Gebühr, bis an ihr Lebensende weiter mit Scheinen versorgt werden können. Nachdem die Personalisierung von Schulen auf der Ebene der Verfahren ihre Bedeutung verloren hat und durch detaillierte und kritische Forschung ersetzt wurde, ist es schon bemerkenswert, wie nun unter dem Label der störungsspezifischen Therapie «Gurus» und «innere Zirkel» mit der Forderung neu aufleben, dass ihre Therapie nur von Personen erbracht werden könne, die eine (kostspielige und bescheinigte) Ausbildung an «ihren» Instituten und bei Personen, die den Segen des Gründers haben, absolviert haben. Und es ist verdächtig, wie mancherorts neue Psychotherapiegesellschaften, natürlich mit angeschlossenen Ausbildungsinstituten, wie die Pilze aus dem Boden sprießen. Da ich selbst in einem Ausbildungsinstitut mitarbeite, frage ich mich, warum ich in dieser Debatte pro und contra Psychotherapieschulen nicht den Contra-Artikel geschrieben habe.

\section{Zusammenfassung}

- Psychotherapieschulen definieren die theoretischen Konstrukte, d.h. die Sprache, mit der Therapeut und Patient unterschiedlichste Probleme und Störungen beschreiben, erklären und ändern können.
- Schulenspezifität beinhaltet auch Evidenzbasierung und Fortentwicklungen in Theorie und Praxis.

- Schulenspezifität beinhaltet auch eine störungsspezifische Psychotherapie.

- Psychotherapieschulen definieren und beschreiben Basisinterventionen und generelle Regeln des therapeutischen Verhaltens

- Therapeuten tun besser daran, ein Theoriesystem, d.h. ein Verfahren richtig zu lernen, als mehrere unzureichend.

- Fachkundenachweise sollten auf der Ebene der Verfahren gefordert werden; eine Unterdifferenzierung kann zu Fehlentwicklungen ohne besseren Patientenschutz führen.

- Es ist von Vorteil, verschiedene Schulen und Behandlungsansätze zu haben. Ein Unitarismus wäre eine Verarmung therapeutischer Optionen.

Michael Linden, Berlin

\section{Literatur}

Brumund L, Märtens M: Die 14 Heilfaktoren der Integrativen Therapie und ihre Bedeutung im Urteil der Therapeuten. Gestalt Integration 1998;2:448-466.

Fromm E: The Art of Loving. New York, Harper Collins, 1974.

GeBA, Gemeinsamer Bundesauschuss der Ärzte und Krankenkassen: Beschluss des Gemeinsamen Bundesausschusses über eine Änderung der PsychotherapieRichtlinien: Definition Verfahren, Methode, Technik, vom 20. Dezember 2007. BAnz Nr 45, 20.03.2008, p 1015.

Grawe K: Grundriss einer Allgemeinen Psychotherapie. Psychotherapeut 1995;40: $130-145$.

Groeben N, Westmeyer H: Kriterien psychologischer Forschung. Weinheim, Juventa, 1975

Helmchen H, Linden M, Rüger U: Psychotherapie - Bedürfnis, Angebot und Bedarf; in Helmchen H, Linden M, Rüger U (Hrsg): Psychotherapie in der Psychiatrie. Berlin, Springer, 1982, pp 1-10

Lieberei B, Linden M: Unerwünschte Effekte, Nebenwirkungen und Behandlungsfehler in der Psychotherapie. Z Evidenz Fortbildung Qualität Gesundheitswesen 2008;102:558-562.

Linden M, Langhoff C, Milev D: Das Mehrebenen-Modell psychotherapeutischer Kompetenz. Verhaltenstherapie 2007a;17:52-59.

Linden M, Langhoff C, Rotter M: Definition, operationalization, and quality assurance of psychotherapy. An investigation with the behaviour therapy-competence-checklist (BTCC). Psychiatria Danubina 2007b;19:308-316.

Linehan M: Cognitive Behavioral Treatment of Borderline Personality Disorder. New York, Guilford, 1993.

McCullough JP: Treating Chronic Depression with Disciplined Personal Involvement: CBASP. New York, Springer, 2006.

Norcross JC, Goldstein MR (Eds): Handbook of Psychotherapy Integration. New York, Basic Books, 1992.

Petzold HG, Märtens M: Wer und was wirkt wie in der Psychotherapie? Mythos «Wirkfaktoren» oder hilfreiches Konstrukt? Integr Ther 1998;1:98-110.

PsychThG, Psychotherapeutengesetz: Gesetz über die Berufe des Psychologischen Psychotherapeuten und des Kinder- und Jugendlichenpsychotherapeuten vom 16. Juni 1998, BGBl. I S. 1311

Rogers CR: On Becoming a Person. New York, Houghton Mifflin Harcourt, 1961 Schippan B, Baumann K, Linden M: Weisheitstherapie. Kognitive Therapie der Posttraumatischen Verbitterungsstörung. Verhaltenstherapie 2004;14:284-293.

Schramm E: Interpersonelle Therapie. New York, Schattauer, 1998.

Sponsel R: Handbuch Integrativer Psychologischer Psychotherapie. Erlangen, IEC, 1995.

WBP, Wissenschaftlicher Beirat Psychotherapie: Verfahrensregeln zur Beurteilung der wissenschaftlichen Anerkennung von Methoden und Verfahren der Psychotherapie. Fassung vom 9.7.09, www.wbpsychotherapie.de/downloads/Methodenpapier27.pdf. 


\section{Contra}

\section{Störungsorientierte Psychotherapien bereichern die therapeutischen Möglichkeiten und steigern die Effektivität}

Ausbildung, klinische Anwendung und Forschung in der Psychotherapieszene sind jahrzehntelang von Psychotherapieschulen wie der Psychoanalyse, Kognitiven Verhaltenstherapie, Gesprächspsychotherapie, Gestalttherapie oder Systemischen Therapie bestimmt worden. Entsprechend wird in Deutschland das gesamte von den gesetzlichen Krankenkassen finanzierte ambulante Behandlungsangebot exklusiv durch die beiden Therapieschulen Psychoanalyse/Tiefenpsychologie und Verhaltenstherapie bestimmt. Es wird jedoch zunehmend Kritik an diesem «Schulendenken» laut. Insbesondere bestehen massive Zweifel, ob es klug war, vor 10 Jahren auch das Psychotherapeutengesetz an Schulen auszurichten, d.h. festzulegen, dass die Ausbildung der Psychologen entweder an einem verhaltenstherapeutischen oder einem tiefenpsychologisch-psychoanalytischen Institut erfolgen muss. Der Gemeinsame Bundesausschuss (GeBA) hat deshalb bereits begonnen, das «Schulendiktat» aufzuweichen, indem er mit dem Konstrukt der «Methoden» in Zukunft auch Psychotherapien für spezifische Störungsbilder, die sich nicht den bisher anerkannten Schulen zuordnen lassen, als genehmigungsfähige Psychotherapieleistungen anerkennen will. So befindet sich zurzeit die IPT nach Klerman und Weissman [1984, 1993] in der Prüfung. Sie stellt ein besonders problematisches Beispiel für die Begrenzung der kassenärztlichen Vergütung auf Schulen oder, wie es offiziell heißt, «Verfahren» dar. Obwohl die Wirksamkeit der IPT durch randomisierte, kontrollierte Studien (RCTs) extensiv belegt ist, wird sie von den Kassen bisher nicht vergütet, da sie keinem der beiden Richtlinienverfahren zugeordnet werden kann. Im Folgenden stelle ich das von mir favorisierte Konzept der störungsspezifischen Psychotherapien in Abgrenzung zu den schulengebundenen Psychotherapien dar.

Traditionell nehmen als «Schulen» bezeichnete Psychotherapieansätze generelle Wirksamkeit bei psychischen und psychosomatischen Störungen in Anspruch. Bei den anerkannten Richtlinienpsychotherapien muss also das allgemeine Wirkprinzip der Schule von Störung zu Störung nicht variiert werden, da man von einer (mehr oder weniger) universellen Wirksamkeit ausgeht. Hat sich eine Therapieschule für drei Störungsbilder, z.B. Depressionen, Angsterkrankungen und Somatisierungsstörungen, empirisch als wirksam erwiesen, wird sie auch für alle anderen psychischen und psychosomatischen Erkrankungen, z.B. Psychosen, Drogenabhängigkeit, Persönlichkeitsstörungen oder Zwangserkrankungen, als Therapieverfahren zugelassen.

Unter anderem die umfangreiche Analyse zur Evaluationsforschung von Grawe und Mitarbeitern [1994] hat ver- deutlicht, dass in Frage gestellt werden muss, ob die behauptete, meist universelle Wirksamkeit der Therapieschulen empirisch Substanz hat. Diese Skepsis beruht auf den bislang ungenügenden Forschungsbestrebungen zur Differenzialindikation der einzelnen Verfahren. Der Status der Psychoanalyse als Richtlinienverfahren für das Gesamtspektrum psychischer und psychosomatischer Störungen basiert sogar auf dem weitgehenden Fehlen von RCTs, d.h. es können auch keine negativen Ergebnisse gegen das Verfahren angeführt werden. Hinzu kommt, dass ein Großteil der Psychotherapierichtungen auf empirisch nicht oder nur zum Teil belegten Theorien basiert. Dies gilt z.B. für die Triebtheorie oder die Selbstkonzepte psychoanalytischer Verfahren, aber auch für theoretische Modelle Kognitiver Therapien, die keineswegs stringent aus der Kognitiven Psychologie abgeleitet sind. Außerdem betrifft dies das Persönlichkeitskonzept der Gesprächspsychotherapie, dessen Vertreter seit Jahren darum kämpfen, dass dieser Ansatz ebenfalls als generell anwendbares Verfahren anerkannt wird.

Weitere Kritik richtet sich gegen den anhaltenden Widerstand vieler Schulen, andere Therapiemethoden und vor allem Elemente anderer Psychotherapierichtungen systematisch und dem gegenwärtigen Forschungsstand entsprechend zu integrieren. Dies, obwohl gewichtige Evidenzen vorliegen, dass die Unterlassung zusätzlicher Strategien bei einigen Erkrankungsformen negative Folgen für den Patienten haben kann. So ist z.B. die Überlegenheit einer Kombinationstherapie im Vergleich zu einer reinen Pharmako- oder Psychotherapie bei chronischen Depressionen [Keller et al., 2000] und älterer depressiver Patienten empirisch gut belegt [Reynolds et al., 1999]; bei schweren Depressionen dürfte sie einem systematischen Review zufolge ebenfalls wirksamer sein als reine Psychotherapie [z.B. Geddes und Butler, 2002]. Gleiches gilt für die Behandlung von Patienten mit schweren Zwangserkrankungen, bei denen Zwangsgedanken im Vordergrund stehen [Kordon et al., 2009]. Bezüglich Psychotherapien ist die Unterlassung einer Expositionsbehandlung bei Zwangserkrankungen ebenso als kritisch zu beurteilen wie eine Konfrontationstherapie (innere Rekonstruktion des Traumas) bei Patienten mit posttraumatischer Belastungsstörung (PTBS) [Frommberger et al., 2009]. Psychotherapeuten, die sich vornehmlich an einer Schulrichtung orientieren, gründen ihr Vorgehen oft nicht systematisch auf aktuelle wissenschaftliche Forschungsergebnisse zur Genese von und Therapiewirksamkeit bei bestimmten Störungsbildern. Es konnte z.B. nachgewiesen werden, dass Verlusterlebnisse, chronische Partnerschaftskonflikte, neue Rollenanforderungen und defizitäre soziale Kompetenzen das Auftreten und die Aufrechterhaltung depressiver Störungen entscheidend bestimmen [Berger et al., 2009]. Im Gegensatz zur störungsspezifischen Psychotherapie suchen Psychotherapieschulen primär nach den von ihrer Theorie favorisierten Problembereichen, z.B. Objektbeziehungsstörung, orale Fixierung, Triebkonflikt, lerntheoretisch begründete kognitive Verzerrung oder operante Fehlkonditionierung. 
Was die dargestellten allgemeinen Wirkfaktoren der Psychotherapie angeht, besteht häufig keine Ausgewogenheit, beispielsweise herrscht bei den psychodynamischen Therapieformen oft eine Überbewertung der Aspekte Klärung, Konfrontation und Deutung gegenüber Problembewältigung und Ressourcenaktivierung vor. Bei der Verhaltenstherapie ist es umgekehrt, d.h. die Symptombewältigung dominiert gegenüber einer Klärung der Symptomentstehung im biographischen Kontext und deren Auswirkung auf die jetzige Therapeut-Patient-Beziehung.

Bei vielen Therapierichtungen war also lange Zeit kein Bezug zu erkennen zwischen den psychotherapeutischen Interventionen und den spezifischen Anforderungen des konkreten Krankheitsbildes. Psychoanalytische Behandlungsmethoden wandten die Technik der Übertragung und Gegenübertragung sowie der Widerstandsdeutung auf alle möglichen psychischen Störungen an, ohne dass störungsspezifische Elemente wie spezifische Krankheitsaufklärung oder spezifische Strategien zur Symptombewältigung erkennbar waren. In der Verhaltenstherapie wurden hingegen z.B. soziale Defizite bei depressiven Patienten ähnlich behandelt wie soziale Defizite bei Angsterkrankungen oder Persönlichkeitsstörungen, und zwar unter der Annahme, dass soziale Defizite bei affektiven Störungen denen bei anderen Störungsbildern entsprächen. Zwar ist inzwischen eine deutlich stärkere Beachtung der ICD- bzw. DSM-Diagnostik und der sich daraus ergebenden differenzialtherapeutischen Konsequenzen zu erkennen, doch sollte dieser Prozess forciert werden.

\section{Störungsorientierte Psychotherapien}

Die international wohl wichtigste Entwicklung der letzten Jahre ist die empirisch fundierte, auf die Charakteristika des konkreten Krankheitsbildes bezogene Ausdifferenzierung therapeutischen Vorgehens. Dies erfordert die genaue Charakterisierung der Krankheitsmerkmale, eine diagnostische Festlegung und daraus abgeleitet spezielle psychotherapeutische Interventionen. Gute Beispiele sind etwa das CBASP für chronische Depressionen [McCullough, 2000] oder die DBT [Bohus, 2002] sowie die Schematherapie für BorderlineStörungen [Giesen-Bloo et al., 2006], die ganz auf diese Störungsbilder zugeschnitten sind. Wegen des erweiterten Spektrums therapeutischer Strategien, d.h. intensivierte biographische Arbeit, differenzierte Bearbeitung der Patient-Therapeut-Beziehung und emotionsinduktive Methoden, kann man sie als Beispiele der Dritten Welle der Verhaltenstherapie bezeichnen [z.B. Öst, 2008]. Mit störungsspezifischen Psychotherapieformen wird das Ziel verfolgt, Psychotherapiemodule für verschiedene Problembereiche, die durch die spezielle Symptomatik, Lerngeschichten, Psychodynamik, innerpsychischen Konflikte und zwischenmenschlichen Problemkonstellationen des jeweiligen Krankheitsbildes hervorgerufen werden, zu nutzen und auf diese anzuwenden. Dabei werden unideologisch und pragmatisch bisherige Schulengrenzen überschritten, d.h. es werden Bausteine anderer Schulen, wenn sie für das spezielle Störungsbild opportun erscheinen und sich in empirischer Forschung als wirksam erweisen, übernommen und für das Störungsbild modifiziert (z.B. proaktive Übertragungsarbeit beim CBASP).

Ein besonders eindrucksvolles Beispiel für die Entwicklung einer störungsspezifischen Psychotherapie stellt die Behandlung essgestörter Patientinnen dar, die in der Regel eine Reihe von therapierelevanten Problembereichen aufweisen: eine Störung der enterozeptiven und emotionalen Wahrnehmung, gewichtsphobisches Verhalten, dysfunktionale Gedanken und Überzeugungen, Autonomie-Abhängigkeits-Konflikte, Sexualängste [Fichter, 2009]. Die störungsspezifische Therapie bei Patienten mit Anorexia nervosa setzt sich aus einem Gesamtbehandlungsplan aus Ernährungsberatung, Körpertherapie, eventuell Pharmakotherapie (z.B. bei einer sekundären Depression), Ergotherapie und beruflicher Belastungserprobung und Rehabilitationsansätzen zusammen. Die Psychotherapie wird krankheitstypische Konflikte wie Abhängigkeits-Autonomie-Konflikte, Körperschemastörungen, Selbstwertprobleme und Sexualängste thematisieren müssen.

Eine solche störungsorientierte Sichtweise bedeutet nicht, dass man die individuellen Erfordernisse und Bedürfnisse des einzelnen Patienten außer Acht lässt, geht aber davon aus, dass die Erkrankung zu einem Zustand führt, in dem Individuen mit prämorbid ganz unterschiedlichen Charakterzügen und Eigenschaften große Ähnlichkeiten im Verhalten, emotionalen Erleben und in kognitiven Abläufen aufweisen (Abb. 1). So zeigen z.B. depressive Patienten im Zustand der Depression viele Übereinstimmungen hinsichtlich Psychomotorik, Mimik, affektiver Gestimmtheit, kognitiven Verzerrungen, somatischen und psychovegetativen Symptomen etc. Gleiches gilt für viele andere Diagnosegruppen. Ohne diese interindividuell ähnlichen psychopathologischen Charakteristika einer akuten psychischen Erkrankung wäre eine Klassifikation und Diagnostik psychischer Störungen nicht möglich. Störungsspezifische Psychotherapie setzt außer an den individuellen Ansatzpunkten des Patienten an genau diesen durch das Störungsbild verursachten Problembereichen an. Mit Abklingen des akuten Zustandsbildes löst sich auch der Bereich großer Ähnlichkeiten in der akuten Krankheitsphase auf und macht wieder einer stärkeren Individualisierung Platz, die veränderte psychotherapeutische Strategien erfordert. Dieses Phänomen hängt mit der Schwere der Symptomatik eng zusammen, d.h. je gravierender das Krankheitsbild ist, umso mehr «nivelliert» es die Patienten und macht zumindest in der Akutbehandlung ein klar störungsorientiertes Vorgehen

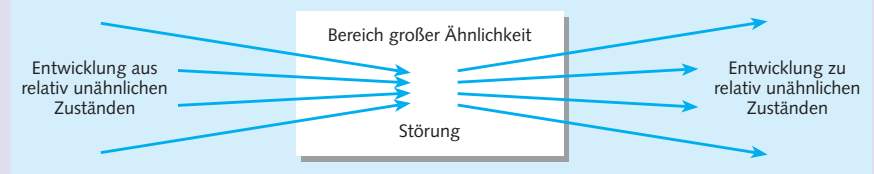

Abb. 1. Angleichung individueller Eigenschaften durch das Störungsbild im Zeitverlauf [nach Caspar, 2000, p 155]. 
sinnvoll. Bei der Therapie weitgehend gebesserter Störungsbilder ist die Störungsorientierung weniger relevant, weil hier oft Aspekte der Selbsterfahrung, der Beziehungsklärung oder des Stressmanagements im Vordergrund stehen.

Um die Besonderheit störungsorientierter Psychotherapie $\mathrm{zu}$ verdeutlichen, sollen zunächst die vier Hauptdeterminanten für die Strukturierung des therapeutischen Prozesses dargestellt werden (Abb. 2).

1. Individuelle Faktoren des Patienten: Relevant sind die Lebensgeschichte, das Alter, die Fähigkeiten und die Persönlichkeit, das Beziehungsverhalten, die aktuelle Lebenssituation, die Lebens- und Therapieziele sowie die Motivation zur Behandlung.

2. Psychotherapieimmanente Faktoren: Für den Therapieprozess ist unter anderem die Wahl des Settings und die Art der therapeutischen Beziehung entscheidend. Ferner hängt der Erfolg von der Kompetenz der Therapeuten ab, d.h. ihrer Erfahrung, welche Verfahren sie gelernt haben und wie sie diese einsetzen.

3. Das gegenwärtige Krankheitsbild: Es bestimmt mit seinen speziellen kognitiven, affektiven, motorischen und somatischen Einschränkungen ebenfalls die Erfordernisse und den Ablauf der Therapie. Spezielle Krankheitsmerkmale wie schwere Körperschemastörungen bei Anorexie oder Suizidalität bei affektiven Störungen erfordern beispielsweise spezielle Interventionen.

4. Therapie-Zeitpunkt: Der Zeitpunkt der Therapie bezüglich des Alters des Patienten und im Verlauf einer Störung sind zusätzlich für das psychotherapeutische Vorgehen entscheidend. Es ist von Bedeutung, ob es sich um einen episodischen Verlauf mit zu erwartendem spontanem Abklingen der Erkrankungsphase - wie bei der Depression - oder um ein chronisches Krankheitsbild ohne Tendenz zur Spontanremission - wie bei einer Zwangsstörung - handelt. Außerdem ist das gegenwärtige Erkrankungsstadium entscheidend, d.h. ob der Patient in einer Akutphase, in der Erhaltungsphase oder in der Phase der Rückfallprophylaxe zur Therapie kommt. All diese Aspekte entscheiden, was für eine Therapie indiziert ist, Kurz- oder Langzeittherapie, in welcher Frequenz und mit welchen Zielen. Während Schulen etwa diesen letzten Aspekt kaum systematisch berücksichtigen, ist die Strategie störungsorientierter Verfahren meist an den Anforderungen der unterschiedlichen Krankheitsphasen orientiert.

Die Akutphase bedingt in der Regel ein Vorgehen mit folgenden Kernelementen: spezielle Gestaltung der therapeutischen Beziehung durch die Charakteristika des speziellen Krankheitsbildes wie z.B. Depression, PTBS, Suchterkrankung oder Borderline-Störung; psychoedukative Elemente für Patienten und deren Angehörige und Darstellung eines entsprechenden Krankheitsmodells und Therapierationals; Behandlungsvertrag/Absprache mit individueller Zielsetzung; Kombination mit weiteren störungsorientierten Interventionen (z.B. medikamentöse Behandlung oder Soziotherapie); Abbau selbst- und therapieschädigenden Verhaltens (z.B.

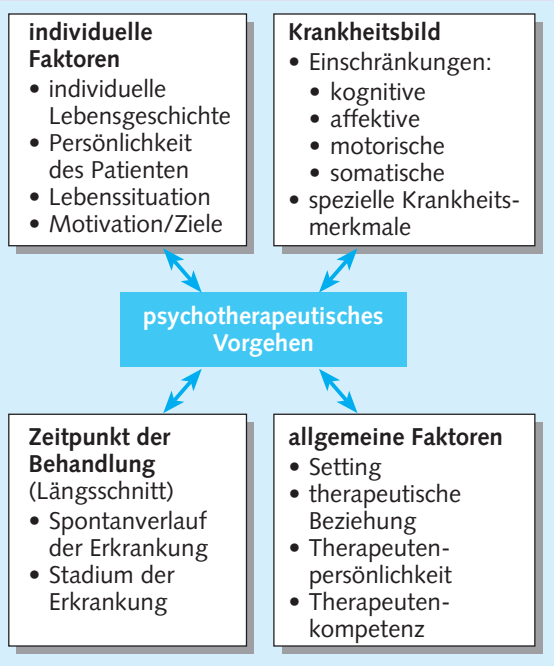

Suizidalität); Sicherung der Compliance; Analyse der Symptomgenese vor dem biographischen Hintergrund; Symptomreduktion durch störungsspezifische Interventionen (z.B. Reizkonfrontationstechniken, Symptommanagement; Aufbau von Alternativverhalten und Reduktion krankheitsaufrechterhaltender Faktoren. In der Akutphase der Erkrankung geht es vor allem darum, die Krankheitssymptome des Patienten zu reduzieren (Bewältigung). Dies erfolgt durch Interventionen, die gezielt die beeinträchtigenden Symptome beeinflussen. Dies kann bei depressiven Erkrankungen Aktivitätsaufbau und gezielter Einsatz positiver Verstärkung sowie Modifikation depressionstypischer kognitiver Verzerrungen sein, während bei Panik-, Zwangs- oder phobischen Störungen Reizkonfrontationstechniken eingesetzt werden. Parallel zur Symptomreduktion wird Alternativverhalten aufgebaut, das die spezielle Symptomkonstellation und den psychosozialen Kontext ebenfalls berücksichtigen muss.

Während der Erhaltungsphase steht in der Regel im Vordergrund, dem Patienten mit einem Selbstmanagementansatz $\mathrm{zu}$ ermöglichen, die Symptomreduktion beizubehalten und das Alternativverhalten $\mathrm{zu}$ festigen. In dieser Phase gewinnt die Bearbeitung krankheitsaufrechterhaltender Faktoren mehr Bedeutung. Die Zielsetzungen dieser Behandlungsphase werden sich bei Suchterkrankungen, Essstörungen, Depressionen oder PTBS sehr unterscheiden; daher ist auch hier eine notwendige Passung auf das Störungsbild unumgänglich.

Während der Phase der Rückfallprophylaxe geht es in der Regel um eine Sensibilisierung für Frühsymptome des speziellen Krankheitsbildes, damit ein drohender Rückfall in Belastungssituationen rechtzeitig erkannt und entsprechend behandelt werden kann. Außerdem dominiert weiterhin die Bearbeitung krankheitsaufrechterhaltender Faktoren sowie die Bearbeitung von Lebensplanung und Stressmanagement.

Zum «Stages of Change»-Modell [Prochaska und Norcross, 2002], das für verschiedene Stadien unterschiedliche Techniken und Arten der Beziehungsgestaltung nahelegt, liegen inzwischen zahlreiche Untersuchungen vor. Sie deuten 
darauf hin, dass Therapieergebnisse stark dadurch bestimmt werden, ob Therapeuten die Erfordernisse des Stadiums, in dem ein Patient mit einem bestimmten Problem gerade steht, gut treffen. Das Modell macht auch noch einmal deutlich, dass mit optimaler Wirksamkeit bei einem breiten Spektrum von psychischen Störungen nur gerechnet werden kann, wenn Therapeuten konzeptuell, methodisch und interaktionell viel Flexibilität und Kompetenz für das jeweilige Störungsbild aufweisen.

\section{Schlussbemerkung}

Störungsorientierte Psychotherapien zielen darauf ab, krankheitsbedingten Erfordernissen gerecht zu werden, ohne die individuellen Erfordernisse/Bedürfnisse des Patienten $\mathrm{zu}$ vernachlässigen. Hieraus ergeben sich bereicherte psychotherapeutische Möglichkeiten und Effektivitätssteigerungen (zumindest bei schweren Krankheitsbildern). Wie in der aktuellen Weiterbildungsordnung des Facharztes für Psychiatrie und Psychotherapie bereits realisiert, sollten in der Aus- und Weiterbildung für Ärztliche und Psychologische Psychotherapeuten zuerst allgemeine Psychotherapieprinzipien vermittelt werden - wozu auch eine Einführung in die wichtigsten tradierten Schulen gehört. Anschließend sollte eine detaillierte Aus- bzw. Weiterbildung in der Behandlung der wichtigsten Störungsbilder, und zwar störungsspezifisch auf dem Boden der bestehenden empirisch gesicherten Forschungsergebnisse erfolgen. Das heißt, jeder ausgebildete Therapeut sollte mit dem bisher am besten belegten Therapieverfahren für akute und chronische Depressionen, Angsterkrankungen, Zwangsstörungen, Essstörungen, Suchterkrankungen, PTBS, Somatisierungsstörungen und Persönlichkeitsstörungen vertraut sein. Hierzu sind eine Befreiung der Curricula von theoretischem Ballast, eine wesentlich stärkere Ausrichtung an den speziellen Krankheitslehren und praktische Therapietechniken erforderlich. Wie bei Kindt und Berger [2009] ausgeführt, sollte der bisher sehr große Zeitaufwand für eine von der Patientenarbeit losgelöste Selbsterfahrung entsprechend der jeweiligen Schulrichtung zugunsten einer deutlichen Ausweitung der Supervision reduziert werden. Die Selbsterfahrung sollte dazu diesen, eigene problematische Charakteristika zu erkennen, die bei der Behandlung von Patienten mit unterschiedlichen Störungsbildern hinderlich und erfolgsmindernd sein können.
Es ist zu hoffen, dass der Schritt des GeBA zu Psychotherapiemethoden den ersten Schritt von den Schulen zu einer Allgemeinen und Speziellen Psychotherapielehre einläutet.

\section{Mathias Berger, Freiburg i.Br.}

\section{Literatur}

Berger M, van Calker D, Brakemeier EL, Schramm E: Affektive Störungen; in Berger M (Hrsg): Psychische Erkrankungen. Klinik und Therapie, ed 3. München, Elsevier, 2009, pp 492-576.

Bohus M: Borderline-Störung. Fortschritte der Psychotherapie. Manuale für die Praxis, Band 14. Göttingen, Hogrefe, 2002.

Caspar F: Therapeutisches Handeln als individueller Konstruktionsprozess; in Margraf J (Hrsg): Lehrbuch der Verhaltenstherapie, Band 1, ed 2. Göttingen, Hogrefe, 2000, p 155-166.

Fichter M: Anorektische und Bulimische Erkrankungen; in Berger M (Hrsg): Psychische Erkrankungen. Klinik und Therapie, ed 3. München, Elsevier, 2009, pp 737-760.

Frommberger U, Nyberg E, Angenendt J, Lieb K, Berger M: Posttraumatische Belastungsstörungen; in Berger M (Hrsg): Psychische Erkrankungen. Klinik und Therapie, ed 3. München, Elsevier, 2009, pp 668-687.

Geddes J, Buttler R: Depressive disorders; in BMJ Publishing Group (ed): Clinical Evidence. Mental Health. The International Source of the Best Available Evidence for Mental Health Care. Margate, Thanet, 2002, pp 61-75.

Giesen-Bloo J, van Dyck R, Spinhoven P, van Tilburg W, Dirksen C, van Asselt $\mathrm{T}$, et al: Outpatient psychotherapy for borderline personality disorder: randommized trial of schema-focused therapy vs. transference-focused psychotherapy. Arch Gen Psychiatry 2006;63:649-658.

Grawe K, Donati R, Bernauer F: Psychotherapie im Wandel von der Konfession zur Profession. Göttingen, Hogrefe, 1994.

Hohagen F, Stieglitz RD, Bohus M, Caspar F, Berger M: Psychotherapie; in Berger M (Hrsg): Psychische Erkrankungen. Klinik und Therapie, ed 3. München, Elsevier, 2009, pp 152-229.

Keller MB, McCullough JP, Klein DN, et al: A comparison of nefazodone, the cognitive behavioral-analysis system of psychotherapy, and their combination for the treatment of chronic depression. N Engl J Med 2000;342:1462-1470.

Kindt H, Berger M: Pro und Contra. Selbsterfahrung: Essenziell oder verzichtbar? Neurotransmitter 2009;3:12-14.

Klerman G, Weissman M: New Applications of Interpersonal Psychotherapy. Washington, DC, American Psychiatric Press, 1993.

Kordon A, Wahl K, Hohagen F: Zwangsstörungen; in Berger M (Hrsg): Psychische Erkrankungen. Klinik und Therapie, ed 3. München, Elsevier, 2009, pp 643-666.

McCullough JP: Treatment for chronic depression: cognitive behavioral analysis system of psychotherapy (CBASP). New York, Guilford, 2000.

Öst LG: Efficacy of the third wave of behavioral therapies: a systematic review and meta-analysis. Behav Res Ther 2008;46:296-321.

Prochaska JO, Norcross JC: Stages of change; in Norcross J (ed): Psychotherapy Relationships That Work. Therapist Contributions and Responsiveness to Patients. New York, Oxford University Press, 2002, pp 303-314.

Reynolds CF, Perel JM, Frank E, et al: Three-year outcomes of maintenance nortriptyline treatment in late-life depression: a study of two fixed plasma levels. Am J Psychiatry 1999;156:1177-1181. 